\title{
Extinction of sequential conditioned inhibition
}

\author{
MICHAEL J. DETKE \\ University of Pennsylvania, Philadelphia, Pennsylvania
}

\begin{abstract}
Extinction of inhibition was examined in three pigeon autoshaping experiments. In Experiment 1 , a sequential conditioned inhibitor (CI) lost inhibitory power after extinction trials. In Experiment 2, this loss of inhibition was replicated, and the effect was general to both the original target and a transfer target that was separately trained in an inhibition design. In Experiment 3, two CIs were trained simultaneously and two sequentially, and one of each was extinguished; all were tested simultaneously and sequentially. The results show that sequential testing is a necessary component for observing loss of inhibition. This is not consistent with an actual loss of inhibitory associations. It is suggested that the extinction trials either decrease processing of the CI, or extinguish its excitatory properties, to which some of the inhibition may be conditioned.
\end{abstract}

Over the last 20 years, a good deal of effort has been directed toward investigating the nature of Pavlovian conditioned inhibition. In the paradigm studied most, a conditioned stimulus (CS) signals the delivery of an unconditioned stimulus (US), except when that CS is preceded or accompanied by a conditioned inhibitor (CI). The common result of such an $\mathrm{A}+, \mathrm{BA}-$ design is that $\mathrm{A}$ becomes a conditioned excitor, evoking a conditioned response, whereas $B$ becomes an inhibitor, interfering with the response to $\mathrm{A}$.

Theories of Pavlovian conditioning provide quite a good account of the circumstances that lead to the development of conditioned inhibition. They suggest that B becomes an inhibitor because of its presence at a time when an excitatory $\mathrm{A}$ is nonreinforced and hence undergoes a decrement in its net associative strength. Although the theories differ in detail and in their language, they share this general description of the acquisition of inhibition (see, e.g., Konorski, 1948, 1967; Pearce \& Hall, 1980; Rescorla, 1979; Rescorla \& Wagner, 1972; Wagner, 1981).

Somewhat more problematic has been the development of a description of the circumstances that lead to the loss of inhibition. Most formulations anticipate that if the conditioned inhibitor were separately presented and followed by the US, it would lose its inhibitory power. However, at least one widely used theory, the Rescorla-Wagner model, also anticipates that if the inhibitor were presented

The work presented here was supported by NSF Grant BNS 88-03514 to Robert A. Rescorla. Michael Detke was supported by a Medical Scientist Training Program Fellowship (funded via NIH grant 5-T32-GM07170). Experiments 1 and 2 served as partial fulfillment of the requirements for the MA degree in Psychology at the University of Pennsylvania. The author wishes to gratefully acknowledge the substantial contributions of Robert Rescorla, advisor on the project. Thanks also go to Steven Robbins for his assistance, and to all members of the lab for their helpful suggestions. Correspondence should be addressed to Michael J. Detke, Department of Psychology, University of Pennsylvania, 3815 Walnut St., Philadelphia, PA 19104-6196. alone, its inhibition should become extinguished (Rescorla \& Wagner, 1972; Wagner \& Rescorla, 1972). This rather surprising prediction has by and large been disconfirmed by experiments designed to assess it (e.g., DeVito \& Fowler, 1986, 1987; Pearce, Nicholas, \& Dickinson, 1982; Rescorla, 1982; Witcher \& Ayres, 1984; ZimmerHart \& Rescorla, 1974). In these studies, separate presentations of previously established inhibitors failed to attenuate their power; indeed in some cases, an inhibitor became more powerful as a result of its separate presentation. Various modifications of this and other models have consequently been suggested, in order to remove the discrepancy from the available data.

However, there have been some recent reports of successful extinction of inhibitors by their separate presentation. For instance, Holland (1985) and Holland and Gory (1986), who used conditioned suppression with aversive USs and rat subjects, reported loss of conditioned inhibition. More recently, Robbins (1990) found that separate presentation extinguished both the excitation and the inhibition controlled by an ambiguously trained stimulus in an autoshaping paradigm with pigeon subjects. These experiments differ from each other in many ways, but they share one difference from previous failures to find extinction of inhibition: they all involved sequential, rather than simultaneous, presentation of $B$ and $A$ on the nonreinforced trials of the $\mathrm{A}+, \mathrm{BA}-$ design used to establish inhibition to B. Holland (1985) has argued that such sequential presentation results in a different form of conditioned inhibition, one that is more susceptible to separate nonreinforced presentation.

In the present experiments, the degree to which sequentially trained inhibitors lose their inhibition when they are separately presented was explored further. In an autoshaping preparation, localized visual stimuli signaled food USs, and the observed response consisted of pecking at those stimuli. Experiments 1 and 2 examined the effects of separate presentation of $\mathbf{B}$ after training with a sequen- 
tial A+, B $\rightarrow$ A- design. The potential loss of B's inhibition was assessed by means of its joint presentation with its training excitor, A, and with a transfer excitor. Experiment 3 compared the effects of separate presentation on inhibitors trained in sequential and simultaneous designs. In order to separate the effects of temporal relation on establishing and on testing inhibitors, the assessment of inhibitors was conducted using both sequential and simultaneous presentations with an excitor. The results confirm that separate presentation attenuates the power of otherwise effective inhibitors. However, such attenuation was apparent only under sequential test conditions, suggesting that it reflected diminished exhibition of the inhibition rather than extinction of the inhibition itself.

\section{EXPERIMENT 1}

One goal in the first experiment was to replicate the basic finding of extinction of a sequential CI (Holland, 1985; Holland \& Gory, 1986; Robbins, 1990). A second goal was to examine whether such extinction is specific to the original CS, or whether it transfers to a new target CS. Transfer of the inhibition (i.e., the decrement in responding) would confirm that the $\mathrm{CI}$ was inhibitory and that the decrement in responding to the original target was not based on some other form of discrimination such as configural learning. Likewise, any loss of effectiveness of the CI that was common to both targets would be attributable to a change in the $\mathrm{CI}$ per se, rather than to an inhibitor-target interaction.

To accomplish these goals, the following design was used, which is summarized in Table 1. A target CS was reinforced (i.e., $\mathbf{A}+$ ), while two CIs were presented in sequential combination with the target, without reinforcement (i.e., $\mathrm{X} \rightarrow \mathrm{A}-, \mathrm{Y} \rightarrow \mathrm{A}-$ ). In addition, a control stimulus was presented on its own, also unreinforced (i.e., $\mathrm{Z}-$ ). Subsequently, one of the CIs was repeatedly presented without reinforcement (i.e., $\mathrm{Y}-$ ). A transfer target was also separately trained (i.e., $\mathrm{B}+$ ). Then both CIs and the control stimulus were tested for their ability to decrease responding to both targets. Using a similar design and preparation, Rescorla (1989), though not addressing extinction, did observe inhibition and transfer of the inhibition.

If the intact inhibitor were to decrease responding to both targets, that would constitute evidence for transfer; if the inhibitor that was separately presented did not decrease responding as much as the intact one, that would confirm that extinction training affected inhibition.

\section{Method}

Subjects and Apparatus. The subjects were 16 experimentally naive female Carneau pigeons about 1 year old and maintained at $80 \%$ of free-feeding weight on a diet of Purina Pigeon Grain. They were housed in pairs, with free access to water and grit, on a 14:10-h light:dark schedule.

The apparatus consisted of eight identical chambers, $27 \times 27$ $\times 35 \mathrm{~cm}$ tall. The metal front wall of each had a $5 \times 5 \mathrm{~cm}$ food
Table 1

Design of Experiments

\begin{tabular}{|c|c|c|c|}
\hline \multicolumn{3}{|c|}{ Phase } & \multirow[b]{2}{*}{ Test } \\
\hline Acquisition & Discrimination & Extinction & \\
\hline \multicolumn{4}{|c|}{ Experiment 1} \\
\hline $\mathrm{A}+, \mathrm{B}+$ & $\begin{array}{l}\mathbf{A}+, \mathbf{B}+ \\
\mathbf{X} \rightarrow \mathbf{A -} \\
\mathbf{Y} \rightarrow \mathbf{A -} \\
\mathbf{Z}-\end{array}$ & Y- & $\begin{array}{c}\mathrm{A}, \mathrm{B} \\
\mathrm{X} \rightarrow \mathrm{A}, \mathrm{X} \rightarrow \mathrm{B} \\
\mathrm{Y} \rightarrow \mathrm{A}, \mathrm{Y} \rightarrow \mathrm{B} \\
\mathrm{Z} \rightarrow \mathrm{A}, \mathrm{Z} \rightarrow \mathrm{B}\end{array}$ \\
\hline \multicolumn{4}{|c|}{ Experiment 2} \\
\hline$A+, B+$ & $\begin{array}{l}\mathbf{A}+, \mathbf{B}+ \\
\mathbf{X} \rightarrow \mathbf{A}- \\
\mathbf{Y} \rightarrow \mathbf{B}- \\
\mathbf{Z}-\end{array}$ & $\mathrm{Y}-$ & $\begin{array}{c}\mathbf{A}, \mathbf{B} \\
\mathbf{X} \rightarrow \mathbf{A}, \mathbf{X} \rightarrow \mathbf{B} \\
Y \rightarrow A, Y \rightarrow B \\
Z \rightarrow A, Z \rightarrow B\end{array}$ \\
\hline \multicolumn{4}{|c|}{ Experiment 3} \\
\hline At & $\begin{array}{l}\text { A+ } \\
\text { VA- } \\
\text { WA- } \\
X \rightarrow A- \\
\text { Y }- \text { A- }\end{array}$ & $\begin{array}{l}\text { W- } \\
Y-\end{array}$ & $\begin{array}{c}\quad \mathbf{A} \\
\text { VA, V } \rightarrow A \\
W A, W \rightarrow A \\
X A, X \rightarrow A \\
Y A, Y \rightarrow A\end{array}$ \\
\hline
\end{tabular}

Note- $A$ and $B$ are target excitatory conditioned stimuli, whereas $V$, $\mathrm{W}, \mathrm{X}$, and $\mathrm{Y}$ are inhibitors, and $\mathrm{Z}$ is a control stimulus. The stimuli separated by the arrow notation $(\rightarrow)$ were presented sequentially, whereas those together were presented simultaneously. The " +" and " - " notations indicate the respective presence or absence of the unconditioned stimulus.

magazine in its center, $5 \mathrm{~cm}$ above the wire-mesh floor. There were three response keys on the front wall, $20 \mathrm{~cm}$ above the floor and $2.5 \mathrm{~cm}$ in diameter, one in the center and one to each side. Located behind the right key was an IEE in-line projector, with which the target colors yellow and blue were presented on the bottom half of the key, and with which three stimuli, which will be referred to as features, were presented on the top half of the key. One consisted of 1-mm black and 2-mm white lines oriented diagonally, downward to the right; the second consisted of 1-mm black and 1-mm white lines oriented diagonally, upward to the right; the third was solid white. When either half of the key was illuminated, the other half was black. All stimuli were generated by Ektachrome slides of drawings composed of Color-aid artist's paper. The remaining walls and ceiling of the chambers were of clear Plexiglas.

The chambers were in sound- and light-attenuating boxes, and ventilation fans provided background noise of $62 \mathrm{~dB}\left(\right.$ re $\left.20 \mu \mathrm{N} / \mathrm{m}^{2}\right)$. During the sessions, a 6-W bulb mounted on the rear wall of each shell was continuously illuminated, except during the operation of the food magazine, at which time a 6-W bulb was illuminated above the food magazine. The magazine contained Purina Pigeon Grain.

Procedure. The birds were first magazine-trained by placing them in the chambers with the food hoppers up (making grain accessible). After they had eaten for about $20 \mathrm{sec}$, the hopper was lowered. The periods of food access were then gradually shortened to $5 \mathrm{sec}$, and the intervals in between were gradually lengthened to $1 \mathrm{~min}$. On each of the 4 following days, the birds were given $485-\mathrm{sec}$ grain presentations at intertrial intervals averaging $1 \mathrm{~min}$.

In the acquisition phase of training, all birds received simple autoshaping with both yellow and blue keylights. On each of 6 days, they received 18 presentations of each target color, terminating with food availability $(+)$-that is, A+, B+. Here, as throughout, all CSs (and USs) were $5 \mathrm{sec}$ in duration, the mean intertrial interval was $1 \mathrm{~min}$, and trial orders were pseudorandom.

In the discrimination phase, which lasted 24 days, all birds received one session of sequential conditioned inhibition training per day. Each session included five types of trials: 8 each of yellow and blue followed by food availability $(A+, B+), 12$ of one 
feature (a putative $\mathrm{CI}$ ) followed by one of the target colors and nonreinforced $(X \rightarrow A-), 12$ more identical to those but with a second feature $(\mathrm{Y} \rightarrow \mathrm{A}-)$, and 12 of the third feature presented alone as a control $(\mathrm{Z}-)$. The physical stimuli were balanced with respect to assignment as $X, Y$, or $Z$, and on trials on which a feature and a target were presented, the offset of the first and the onset of the second were contiguous.

In the extinction phase, which lasted 6 days, all birds received 50 non-reinforced presentations of $Y$ per day. Food was not available in the experimental chambers at any time during these sessions. The CSs were balanced with respect to which one was extinguished, on the basis of percentage of inhibition during the last 2 days of the discrimination phase.

On the next 2 days, summation test sessions were conducted. They began with two reinforced presentations of each target color, in ABBA order, so as to return the subjects to their previous levels of responding to the targets. Then there were three blocks of the following eight stimuli or stimulus compounds: blue and yellow, preceded by nothing, the control stimulus, the intact $\mathrm{CI}$, or the extinguished $\mathrm{CI}$. The test stimuli were nonreinforced, except for the target colors alone, which were reinforced in the second session to maintain response levels. The orders of the test stimuli were counterbalanced.

\section{Results and Discussion}

The results of the training are summarized in Figure 1. Early in the discrimination phase, the CIs ( $\mathrm{X}$ and $\mathrm{Y}$ ) elicited some responding on their own and enhanced the responding to the original target somewhat. This was probably the result of second-order conditioning, because the targets were followed by food and the inhibitors were followed by the original target. This is a common finding in designs of this sort (see, e.g., Gokey \& Collins, 1980; Rescorla, 1989). Over the course of the discrimination training, though, keylight pecking increased in response to both targets; it gradually decreased in response to all other stimuli and to the target when it followed either CI. The data were pooled over the last 2 days of discrim- ination training and analyzed with a one-factor repeated measures analysis of variance (ANOVA), which showed a reliable effect of which stimuli were present $[F(3,45)=$ $18.038, p<.001]$. Pairwise comparisons with NewmanKeuls's statistic showed that responding was greater to each target presented alone than to the original target when it was preceded by either of the inhibitors $[q s(3 / 4,45) \geq$ $6.49, p s<.01]$.

The results of the 2 days of summation testing are summarized in Figure 2 . These data were analyzed with a twofactor repeated measures ANOVA, in which target (A vs. B) was one factor, and feature (none, $X, Y$, or $Z$ ) was the other.

The most obvious effect was that overall there was greater inhibition in responding to the original target (A) than there was to the transfer target (B). This observation is supported statistically by the reliable main effect of which target was present $[F(1,15)=13.65, p<.005]$.

The second apparent effect is that the features affected the levels of responding to the original target but not the transfer target. In agreement with this observation, the ANOVA revealed a reliable effect of which feature was present $[F(3,45)=3.147, p<.05]$, and a reliable interaction between feature and target $[F(3,45)=6.786$, $p<.005]$.

Pairwise comparisons among the features applied to the original target show that responding was reliably inhibited by the intact $\mathrm{CI}(\mathrm{X})$ in comparison with the control stimulus $(Z)[q(3,45)=6.47, p<.01]$. Additionally, this inhibition was attenuated by the extinction training; responding was greater to the extinguished $\mathrm{CI}(\mathrm{Y})$ than to the intact one $(\mathrm{X})[q(2,45)=2.975, p<.05]$. The effect was not complete; there was still greater inhibition by the extinguished $\mathrm{CI}(\mathrm{Y})$ than by the control stimulus $(\mathrm{Z})[q(2,45)$ $=3.517, p<.05]$.

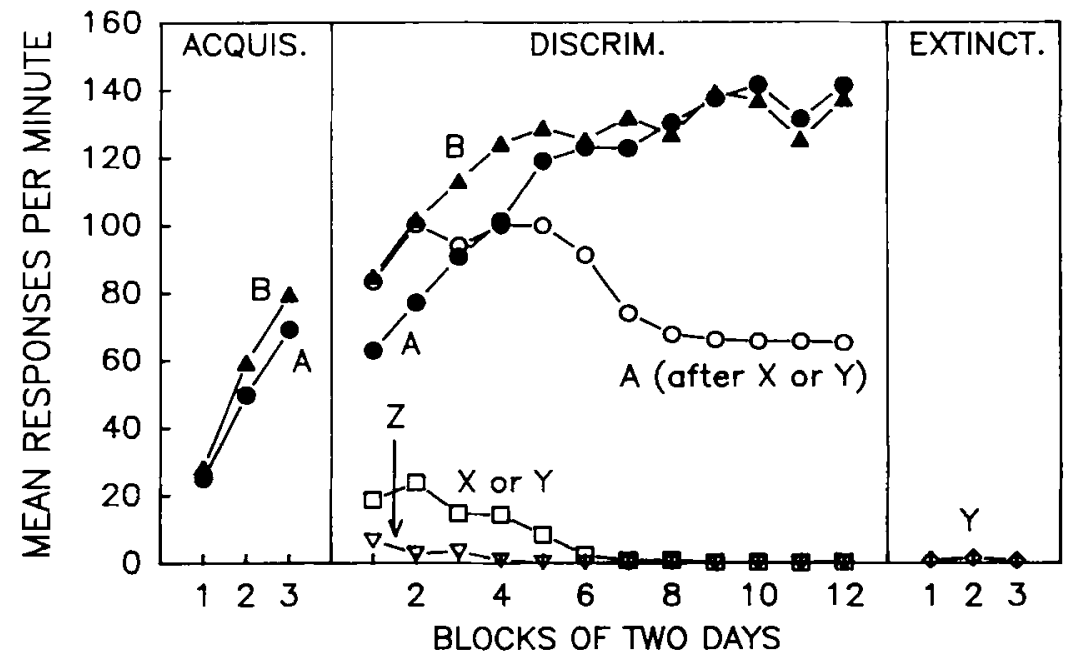

Figure 1. Mean responses per minute during training in Experiment 1, to the target conditioned stimuli ( $A$ and $B)$, to the control stimulus (Z), to $A$ on trials when it was preceded by either conditioned inhibftor ( $A$ after $X$ or $Y$, to the intact and extinguished conditioned inhibitors collapsed $(X$ or $Y$ ), and to $Y$ alone during extinction trials. 


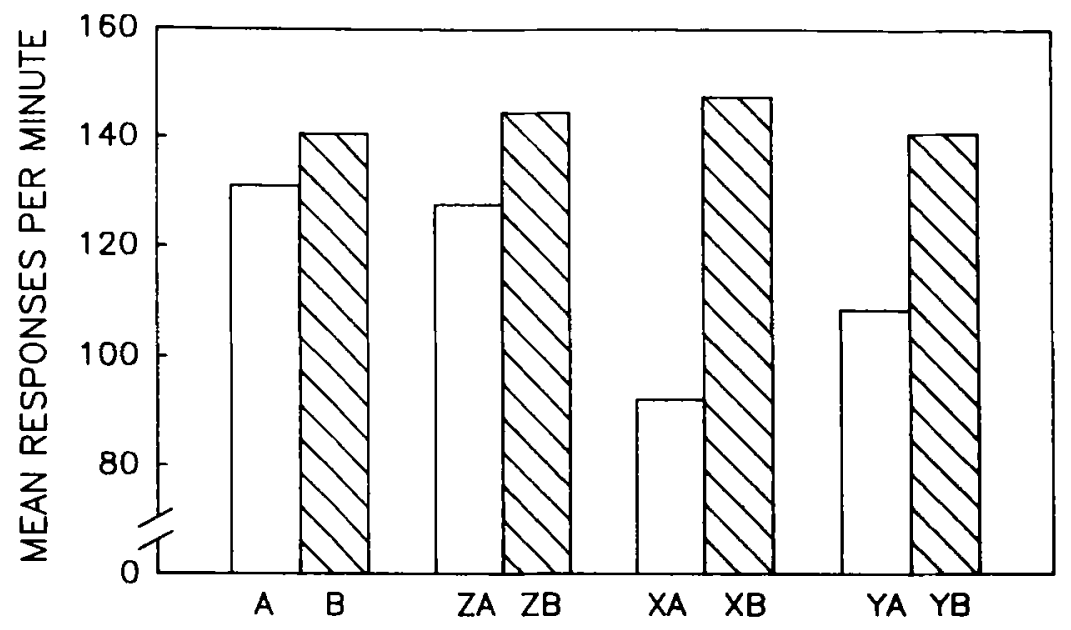

Figure 2. Mean responses per minute to the original (A) and transfer (B) target conditioned stimuli during testing in Experiment 1, when each was presented alone or preceded by the control stimulus $(Z)$, the intact sequential inhibitor $(X)$, or the extinguished sequential inhibitor $(Y)$.

Pairwise comparisons among the features applied to the transfer target (B) showed that there were no reliable differences between the features in their ability to reduce responding to this target.

The primary finding of Experiment 1 was that a sequentially trained $\mathrm{Cl}$ reduced responding to its original target CS, and that this inhibition was attenuated by extinction training. This is a replication of the findings of Holland (1985) and Holland and Gory (1986). It is to be contrasted with a number of failures to see extinction of the inhibition generated by simultaneously trained CIs (e.g., DeVito $\&$ Fowler, 1986, 1987; Pearce et al., 1982; Rescorla, 1982; Witcher \& Ayres, 1984; Zimmer-Hart \& Rescorla, 1974).

However, the inhibition failed to transfer to a separately trained excitatory CS, and thus an extinction effect could not be assessed in this case. This is also consistent with the findings of Holland and Gory (personal communication, February 1990). Given the lack of transfer, one cannot claim that the decrement in responding to the $\mathrm{CI}$ was controlled by the $\mathrm{CI}$ alone. For example, it is possible that it was the result of priming (see, e.g., Wagner, 1978) of the original target by the inhibitors, which would reduce processing of that target. If this were the case, one would not expect that the effect would transfer, and one would expect that the priming, when it was present, would be sensitive to extinction. This is precisely what was observed. Regardless of whether or not priming was the mechanism underlying the lack of transfer, though, the original point remains: one cannot conclude that the inhibition observed was due to the $\mathrm{CI}$ alone. Thus one cannot conclude that the loss of inhibition that was seen was due to a change in the associative strength of the CI alone.

\section{EXPERIMENT 2}

Experiment 2 was an attempt to replicate the inhibition and the extinction of inhibition seen in Experiment 1, and to show that these effects would both transfer to a separate CS. To improve the chances of demonstrating transfer, both targets were embedded in conditioned inhibition designs, which also had the effect of ensuring that the transfer target had some history of nonreinforcement. Both the former (Holland, 1989) and the latter (Rescorla, 1985) have been implicated as being important to the demonstration of transfer.

Therefore, the following design was used in Experiment 2 (it is also summarized in Table 1). Two target CSs were again paired with food (i.e., A+, B+), and two inhibitors were again trained in sequential fashion; but here, one inhibitor was trained with each target (i.e., $\mathrm{X}-\mathrm{A}-, \mathrm{Y} \rightarrow \mathrm{B}-$ ). One inhibitor was later presented repeatedly without reinforcement (i.e., Y-). A fifth stimulus again served as a control, presented without reinforcement (i.e., $\mathrm{Z}-$ ).

\section{Method}

Subjects and Apparatus. The subjects and apparatus were similar to those in Experiment 1.

Procedure. The procedure was the same as in Experiment $\mathbf{l}$ except for the following. During the discrimination phase, one inhibitor was trained with each excitor. So there were five trial types: 8 of each target color followed by food $(\mathrm{A}+, \mathrm{B}+), 12$ of one feature (a putative inhibitor) followed by one of the target colors $\left(X \rightarrow A^{-}\right)$and nonreinforced, 12 of a second feature (also putative inhibitor) followed by the other target color $(\mathrm{Y} \rightarrow \mathrm{B}-)$ and nonreinforced, and 12 of the third feature presented alone as a control stimulus $(\mathrm{Z}-)$. The physical stimuli used as features were balanced in their assignments as $X, Y$, or $Z$, and the physical stimuli used as targets were balanced in their assignments as $\mathrm{A}$ or $\mathrm{B}$. 


\section{Results and Discussion}

The results of the training were virtually identical to those from Experiment 1. Again there was some evidence of second-order conditioning early in the discrimination phase-the inhibitors elicited responding, and they enhanced responding to the targets. And again, over time, keylight pecking increased in response to the targets presented alone and gradually decreased in response to all other stimuli and combinations. The data were pooled over the last 2 days of discrimination training and analyzed with a one-factor repeated measures ANOVA, which showed a reliable effect of which stimuli were present $[F(3,45)=30.70, p<.001]$. Responding was greater to the targets when they were presented alone than when they were preceded by their inhibitors $[q s(3,45) \geq$ 8.46 , ps $<.01]$.

The results of the testing are presented in Figure 3. The general appearance of the data suggests that the features affected responding to both targets similarly. The pattern of effects of the features is like that involving only the original target in Figure 2. Namely, the intact $\mathrm{CI}(\mathrm{X})$ inhibited responding, and the inhibition was attenuated by extinction training; the extinguished inhibitor ( $Y$ ) did not reduce responding as much.

These findings were confirmed by statistical evaluation. Again, a two-factor repeated measures ANOVA was used, again with targets ( $A$ and $B$ ) as one factor, and features (none, $\mathrm{X}, \mathrm{Y}$, and $\mathrm{Z}$ ) as the other. There was a reliable effect of features $[F(3,45)=10.05, p<.001]$, but not of targets $[F(1,15)=.10]$ and there was no interaction $[F(3,45)=1.72]$. Given this, we compared the effects of the features, collapsed across targets. The first finding was that the inhibition was present and transferred. Responding was attenuated more by $\mathrm{X}$ than by the con- trol stimulus $(\mathrm{Z})[q(3,45)=4.79, p<.01]$. The second finding was that the inhibition was attenuated by extinction training. $X$ reduced responding reliably more than $\mathrm{Y}$ did $[q(2,45)=3.4, p<.01]$. The difference between $\mathrm{Y}$ and $\mathrm{Z}$ was not reliable $[q(2,45)=1.35, p>.05]$.

It is noteworthy that in this design, training was carried out with the intact $C I(X)$ preceding target $A$, and with the extinguished $\mathrm{CI}(\mathrm{Y})$ preceding $\mathrm{B}$. Thus, presentations of $X \rightarrow A$ and $Y \rightarrow B$ during the test session were direct tests, measuring inhibition in the same manner as that in which it was trained. Conversely, presentations of $\mathrm{X} \rightarrow \mathrm{B}$ and $\mathrm{Y} \rightarrow \mathrm{A}$ constituted transfer tests. Thus, pairwise comparisons of intact and extinguished $\mathrm{CIs}$ on a given target (e.g., $X \rightarrow A$ vs. $Y \rightarrow A$ ) would not be valid measures of extinction, since they would confound extinction history with transfer testing. More specifically, $X$ may have inhibited responding to $A$ better than $Y$ inhibited responding to $A$ for either or both of two reasons: because $\mathrm{X}$ was not extinguished, or because it was not tested in transfer. Meanwhile, $X$ may have inhibited responding to $B$ better because it was intact, but this effect may have been mitigated by the fact that it was tested in transfer.

There were two primary findings in Experiment 2. The first, extinction of inhibition, amounts to a replication of the findings of Experiment 1. The second, transfer of both the inhibition and the extinction, challenges the alternative account offered for the results of Experiment 1. If the inhibition were mediated by an association between the inhibitor and its original target, as with, for example, priming, one would not expect to see transfer of the inhibition to another target.

However, now that the extinction of sequential inhibition has been replicated and shown to be a function of

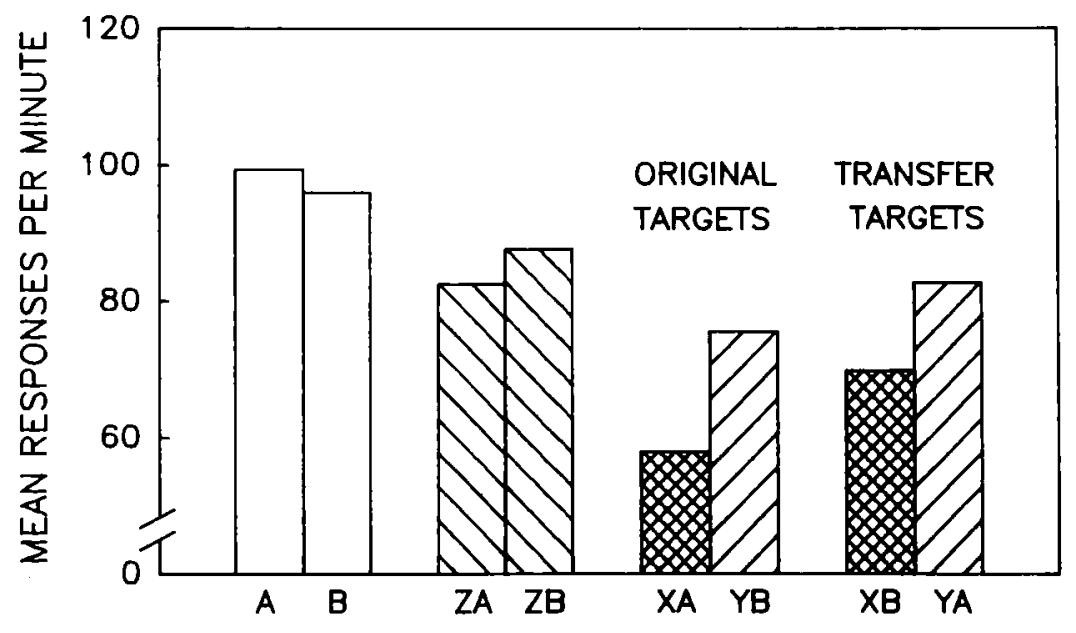

Figure 3. Mean responses per minute to the target conditioned stimull (A and B) during testing in Experiment 2, when each was presented alone or preceded by the control stimulus (Z), the intact sequential inhibitor $(\mathrm{X})$, or the extinguished sequential inhibitor (Y). Note the changed order of XA, YB, XB, and YA, which groups these compounds according to whether or not they were tested in transfer. 
the inhibitor itself, it stands in starker contrast to the fact that simultaneous inhibition is not extinguished. There are several plausible explanations for this discrepancy.

One notion is that the failure to see extinction of simultaneous CI may merely be due to some interaction between the extinction trials and the previous training and/or subsequent testing. Specifically, there may be a failure to generalize from the extinction procedure to the training and/or testing, if a $\mathrm{CI}$ is perceived differently when presented on its own (as in extinction) rather than in simultaneous compound with another stimulus (as in training and testing in a simultaneous design).

Concerning this possibility, Zimmer-Hart and Rescorla (1974, Experiment 4) showed that there was no loss of simultaneous conditioned inhibition, even when the extinction trials involved presentation of the same stimulus compound used in training and testing. And more generally, Holland and Gory (1986) and Rescorla (1989) have shown that simultaneous CIs inhibit responding when tested sequentially, and vice versa. This suggests that features presented in these ways are not seen as qualitatively different. These findings diminish the likelihood that generalization decrement plays a significant role in the failure to see extinction of simultaneous Cls.

A second possibility, put forth by Holland (1985), is that the content of learning is different in simultaneously and sequentially trained CIs. Specifically, Holland suggested that simultaneously trained CIs predicted the absence of the US, and thus, that extinction training led to no new learning. On the other hand, Holland suggested that sequentially trained CIs (or "negative occasion setters") predicted that a CS-US relationship would not hold; for these stimuli, extinction trials represented new information, because no stimuli followed them (rather than a CS without a US). Thus they should be extinguished. Therefore, this account predicts that the manipulation of importance is sequential versus simultaneous training.

A third possibility is that there is an effect of the way the testing is carried out. That is, an inhibitor trained either simultaneously or sequentially may have had some property altered by extinction trials, but may reveal such a change preferentially in one type of testing-such as sequential testing. Or it may be that the change is only revealed when the $C I$ is tested with a specific type of target CS, such as one trained in a sequential conditioned inhibition design.

Only one study has specifically addressed the possibility that the testing method influences extinction of inhibition results. Holland and Gory (1986) used a $2 \times 2$ design (simultaneous or sequential training and simultaneous or sequential testing) and found that training, but not testing, affected the outcome. However, Rescorla (1989), while not addressing extinction of inhibition, did find that it was the testing procedure, not the training, that influenced the inhibition of responding in a similar $2 \times 2$ study. And Holland (1989) has also detected differences in inhibition that were due to the testing procedure, rather than the form of training.

Additionally, in the Holland and Gory (1986) study, training of the CIs was confounded with the training of the targets. That is, one target was used for sequential training $(\mathrm{A}+, \mathrm{X} \rightarrow \mathrm{A}-)$ and testing, and another for simultaneous training $(\mathrm{B}+, \mathrm{YB}-)$ and testing. This leaves open the possibility that the differences found in testing were due to the different treatments of the targets rather than the CIs. One way to rule this out is to employ a training design such as $\mathrm{A}+, \mathrm{XA}-, \mathrm{Y} \rightarrow \mathrm{A}-$ (Rescorla, 1989), which generates one simultaneous and one sequential inhibitor, but a common target.

\section{EXPERIMENT 3}

The goal of Experiment 3 was to evaluate the relative contributions of sequential training and testing to the extinction of inhibition, while controlling for the treatment of the target. To the degree that training is the important variable, this would imply that different forms of learning took place. Conversely, to the degree that the testing method influences the outcome, this would suggest that something aside from the content of learning is involved. And finally, to the extent that both testing and training affect the extinction, it would imply that there is some interaction between the content of the learning and the method of testing.

The following design, which is summarized in Table 1, was used. One target CS was again paired with food, and four CIs were trained, two sequentially and two simultaneously. One sequential $\mathrm{CI}$ and one simultaneous $\mathrm{CI}$ were then extinguished. No control stimulus was employed in this experiment, because no differences had been generated by the control stimuli in the first two experiments, and additional stimuli would have increased the risk that the subjects would fail to discriminate between the CIs. For the same reason, a transfer test was not incorporated into this experiment.

The testing consisted of presenting the target alone, and all four stimuli along with the target, in both simultaneous and sequential fashion. Thus the design is best summarized as $2 \times 2 \times 2$, in that a given inhibitor was simultaneously or sequentially trained, then extinguished or left intact, and then both simultaneously and sequentially tested.

\section{Method}

Subjects and Apparatus. There were 32 subjects, which were similar to those used in the above experiments. The apparatus was also similar, except that there were only four boxes used, and the physical stimuli were different. The target consisted of $2-\mathrm{mm}$ black and $2-\mathrm{mm}$ white lines oriented diagonally $\left(45^{\circ}\right.$ from vertical), downward to the right, projected onto the left side of the key; and the inhibitors were the colors red, green, yellow, and blue projected onto the right side of the key.

Procedure. The procedure was the same as in Experiment 2, except for the changes noted here. 
The experiment was run in two replications; the procedural variations are noted where they occur.

In the acquisition phase of training, all birds received simple autoshaping with the target. On each of 6 days, they received 24 presentations of the target pattern, terminating with food availability $(+)$-that is, At.

The discrimination phase lasted 32 days. During this phase, two inhibitors were trained simultaneously and two were trained sequentially. Thus the five trial types were: 12 of the target pattern followed by food $(A+), 12$ each of two inhibitors presented simultaneously with the target pattern and nonreinforced (i.e., $12 \mathrm{VA}-$, $12 \mathrm{WA}-$ ), and 12 each of two inhibitors followed by the target pattern and nonreinforced (i.e., $12 \mathrm{X}-\mathrm{A}-$ and $12 \mathrm{Y} \rightarrow \mathrm{A}-$ ). The inhibitors were balanced with respect to which physical stimuli were assigned as which of the four inhibitors.

In the first replication, the extinction phase lasted 8 days, on each of which all birds received 30 nonreinforced presentations each of one of the simultaneously trained CIs and one of the sequentially trained CIs (i.e., $30 \mathrm{~W}-, 30 \mathrm{Y}-$ ), for a total of 240 of each. Summation tests were conducted on the next 2 days. In the second replication, all birds received 30 nonreinforced presentations each of $W$ and $Y$ for 7 days and then received 15 nonreinforced presentations each of $W$ and $Y$, followed by summation testing, on the 8th and 9th days. Again there were 240 nonreinforced trials of each extinguished stimulus and 2 days of summation testing, but the testing followed the extinction immediately.

The changes that were incorporated into the second replication were an attempt to minimize any spontaneous recovery of the inhibition in the extinguished inhibitors, by testing them immediately, rather than after a 24-h delay. Robbins (1990) demonstrated extinction of the inhibition controlled by a stimulus in a sequential negative patterning design. He also found spontaneous recovery of this extinction in $48 \mathrm{~h}$, and spontaneous recovery of other forms of learning in as little as $24 \mathrm{~h}$.

The summation test sessions began with four reinforced presentations of the target pattern, in order to retain previous levels of responding to the target. Then there were three blocks of the following nine stimuli or stimulus compounds: the target pattern, each of the four inhibitors in simultaneous compound with the target, and each of the four inhibitors presented sequentially with the target. The test stimuli were nonreinforced, except for the target alone, which was reinforced to maintain response levels.

\section{Results and Discussion}

First, an ANOVA was run using replications as one factor; the main effect of replications was not reliable $[F(1,30)=1.45, p=.24]$, and there were no reliable interactions involving replications $(p s>.05)$. Thus all subsequent analyses were collapsed across replications, and replications will be ignored hereafter.

The results of the training are summarized in Figure 4. There was again some responding to the inhibitors early in training, and there was enhanced responding to $A$ when it was preceded by the inhibitors. Again, this was probably the result of second-order conditioning. Over the course of the discrimination training, though, pecking increased in response to $A$ alone and gradually decreased in response to all other stimuli and combinations. Over the last 2 days of discrimination training, there was a reliable effect of stimuli [one-factor repeated measures ANOVA, $F(2,62)=79.38, p<.001]$. Responding was greater to $A$ presented alone than to $A$ when it was preceded by or compounded with inhibitors $[q s(2 / 3,62)$ $\geq 12.69, p \mathrm{~s}<.01]$

The results of the 2 days of summation testing are summarized in Figure 5. Shown at left is the mean response rate during the target excitor when it was presented alone. To the right are the response rates during that excitor when it was presented in either simultaneous or sequential compound with an inhibitor that had been trained in either a simultaneous or a sequential relation and had either been subjected to extinction or not. These last eight trial types were analyzed with a three-factor repeated measures

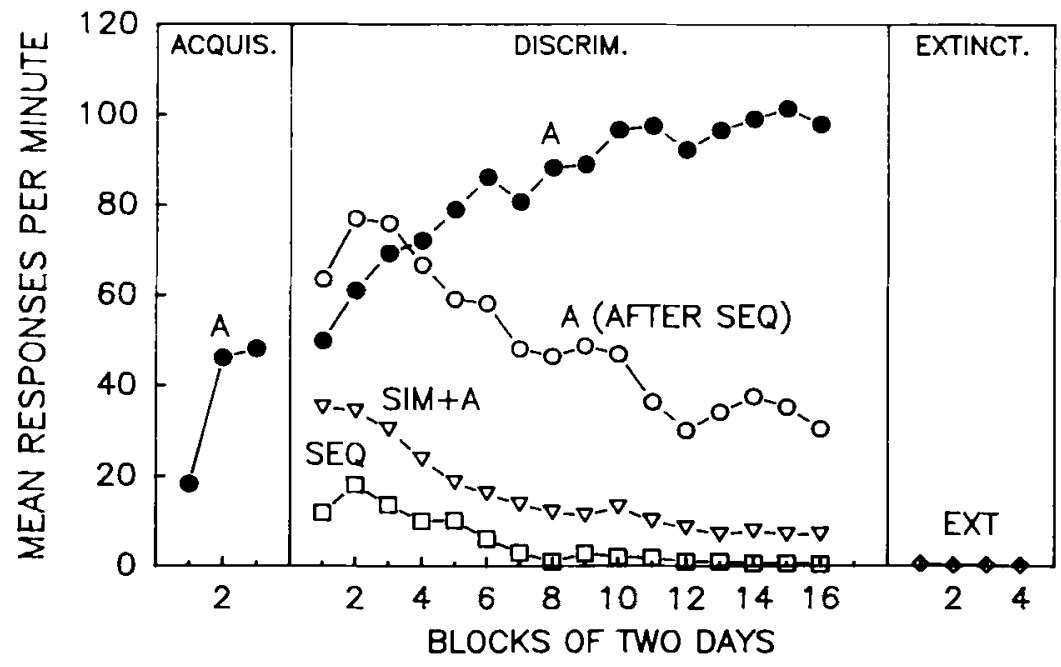

Figure 4. Mean responses per minute during training in Experiment 3, to the target conditioned stimulus (A), to A when compounded with either of the simultaneously trained inhibitors $V$ or $W(S M M+A)$, to $A$ when preceded by either of the sequentially trained inhibitors $X$ or $Y$ (A [AFTER SEQ]), to the sequentially trained inhibitors collapeed (SEQ), and to the extinguished inhibitors $W$ and $Y$ collapsed (EXT). 


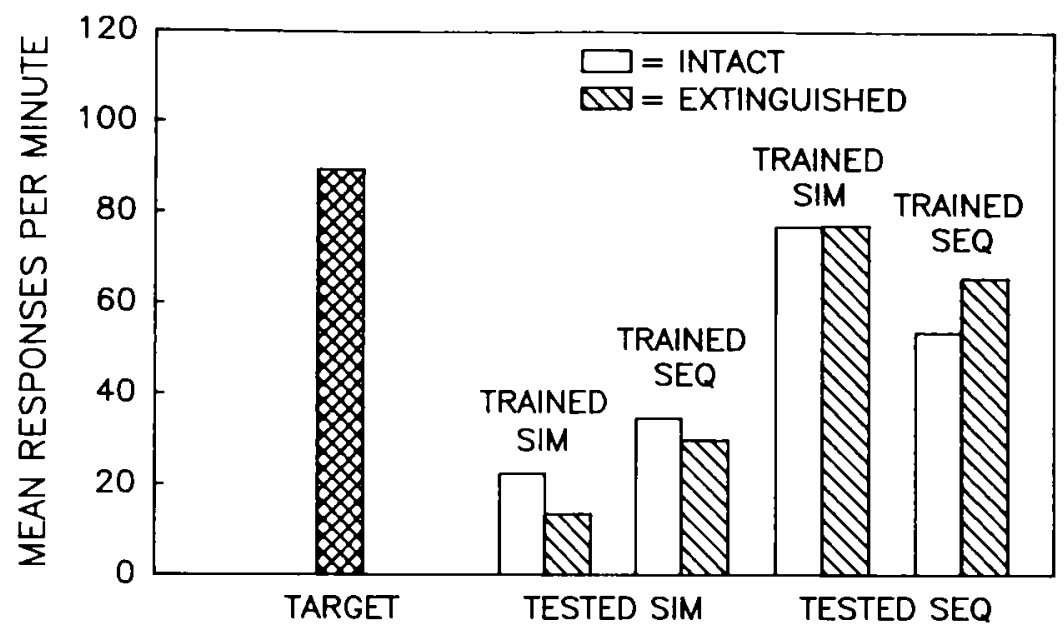

Figure 5. Mean responses per minute to the target conditioned stimulus during testing in Experiment 3, when presented alone, and when tested simultaneously (SIM) and sequentially (SEO) with intact or extinguished inhibitors, which had been trained simultaneously or sequentially.

ANOVA, with training method (simultaneous vs. sequential) as one factor, extinction (intact vs. extinguished) as the second factor, and testing method (simultaneous vs. sequential) as the third.

Inspection of Figure 5 suggests an effect of testing method. Responding to the excitor was substantially higher when a sequential test method was employed. The difference between the response rates in the four bars on the right relative to the four on the left was confirmed by a main effect of testing method in the ANOVA $[F(1,31)=$ $33.57, p<.001]$.

However, the data do not suggest a simple effect of training method. Overall, sequential training did not generate less inhibition than simultaneous training did $[F(1,31)=$ .31]. The data do suggest an interaction between training and testing methods, though. Sequentially trained inhibitors were more effective than simultaneously trained ones when tested sequentially, whereas simultaneously trained inhibitors were more effective than sequentially trained ones when tested simultaneously. This was confirmed statistically by a reliable testing method $\times$ training method interaction $[F(1,31)=15.22, p<.001]$.

Over all other treatments, there was no consistent effect of extinguishing an inhibitor. The ANOVA revealed no main effect of extinction $[F(1,31)=.022]$. Nor was there a reliable interaction between training method and the effects of the extinction manipulation $[F(1,31)=2.29$, $p>.10]$. However, the ANOVA did reveal an interaction between testing method and the effects of extinction $[F(1,31)=4.27, p<.05]$. This demonstrates that sequential testing revealed a greater decrement in inhibition as a result of extinction than did simultaneous testing. Although this is particularly apparent with sequentially trained inhibitors, the absence of a three-way interaction in the ANOVA $[F(1,31)=.66]$ fails to support the claim that training method contributed to the effect.
The small size of the extinction effect in the simultaneously trained, sequentially tested inhibitors might lend to the appearance of a three-way interaction. However, the absence of a larger extinction difference between these two inhibitors may simply be due to a ceiling effect, because the intact inhibitor did not by itself reduce responding to the target greatly. Indeed, similar scale effects, if present, would have biased against the results that were found here for all three other groups: ceteris paribus, CIs producing little inhibition (i.e., at the high end of the scale) would have been less likely to evidence further loss of inhibition than would those with much inhibitory strength (i.e., the ones farther down on the scale, such as the simultaneously tested ones).

Finally, one more (nonreliable) trend probably underlies these data as well; simultaneous training may have led to greater inhibition after extinction than before. Many experimenters have seen greater inhibition after extinction trials when simultaneous training is used (e.g., Zimmer-Hart \& Rescorla, 1974). Cunningham (1981) has suggested that this is because extinction trials lead to the loss of within-compound associations. And it may be the case that stronger within-compound associations form as a result of simultaneous training (as opposed to sequential training). If as this suggests, simultaneous training disposes a CI toward having greater inhibition after extinction, and as we have shown, sequential testing (in comparison with simultaneous testing) evidences less inhibition after extinction, one would expect training, testing, and extinction to interact. And although the data here do not demonstrate the former of these effects, they are certainly consistent with the whole.

In summary, the results of Experiment 3 support two conclusions. The first is that the way in which the testing is carried out can have a powerful effect on the results. The testing method alone influenced the level of inhibition, and the testing method interacted with the training 
method, in that more inhibition was evidenced when the testing was carried out in the same manner as the training. Rescorla (1989) demonstrated the former of these effects and found a (nonreliable) pattern consistent with the latter. These two effects underscore the importance of the testing procedure in general.

The major finding from Experiment 3, however, was that extinction of inhibition is a function of (at least) the testing procedure. More precisely, greater extinction was evidenced when the testing was sequential. Other trends may be obscuring this effect to some degree. One of these may be the loss of within-compound associations, leading to greater inhibition after extinction-particularly in simultaneously trained CIs.

\section{GENERAL DISCUSSION}

The primary finding was that extinction of inhibition is seen as a result of sequential testing. This is not consistent with any model that depicts extinction of inhibition simply as associative loss. Such models would not predict that loss of inhibition would be preferentially evidenced in one type of testing. Rather, they would predict that the $\mathrm{CI}$ should lose its ability to inhibit responding in general.

One fairly straightforward account that is consistent with the present results is that there is a loss of attention to the $\mathrm{Cl}$ as a result of extinction training. The assumption is that this would cause the $\mathrm{CI}$ to be less effective at inhibiting responding in subsequent testing. At least one model would account for such a processing decrement as a result of extinction trials and would predict that it would manifest itself during testing (Mazur \& Wagner, 1982; Wagner, 1981).

But the question remains as to why such an effect is not apparent during simultaneous testing. There are at least two plausible accounts for this. The decrement in processing of the CI may be mitigated by the simultaneous presentation of a target during testing, perhaps because such presentation enhances attentional mechanisms in a general way. It may also be minimized by a generalization decrement, in that the inhibitor is presented by itself in the extinction trials, whereas another stimulus co-occurs during simultaneous testing.

Another account of extinction of inhibition should be considered, however, although it is perhaps not as intuitive. It is clear that at least the sequentially trained CIs gain some excitatory strength early in training, probably through second-order conditioning (see Figure 1). And it is certainly plausible that simultaneously trained CIs also attain some excitatory strength in the same manner, and that both types of stimuli retain some of their excitation. If so, the excitation itself may become a powerful signal that controls inhibition. Ideas of this sort are not altogether new (cf. Lysle \& Fowler, 1985). In support of this notion, it has been shown that training a $\mathrm{CI}$ as an excitor at the same time (i.e., running a negative patterning de- sign) increases the CI's ability to inhibit responding (Rescorla, 1991).

Given that excitation may play some role in controlling the inhibition of responding and that excitation is thought to be lost as a result of extinction training, one might expect some of the control of inhibition to be lost as well. This may be the mechanism that yields extinction of inhibition. And given that excitation (provided by the target) would be present concurrently with the inhibitor during simultaneous testing, one might not expect to find extinction of inhibition during such testing.

In summary, then, the major finding was that extinction of inhibition is evidenced preferentially during sequential testing. This finding is not consistent with models that depict extinction of inhibition as the result of associative loss. Two different accounts are consistent with these data, and the present experiments were not designed to distinguish between them.

\section{REFERENCES}

Cunningham, C. L. (1981). Association between the elements of a bivalent compound stimulus. Joumal of Experimental Psychology: Animal Behavior Processes, 7, 425-436

DEVITo, P. L., Fowler, H. (1986). Effects of contingency violations on the extinction of a conditioned fear inhibitor and a conditioned fear excitor. Journal of Experimental Psychology: Animal Behavior Processes, 12, 99-115.

DeVito, P. L., Fowler, H. (1987). Enhancement of conditioned inhibition via an extinction treatment. Animal Leaming \& Behavior. $15,448-454$.

Gokey, D. S., \& Coluns, R. L. (1980). Conditioned inhibition in feature negative discrimination learning with pigeons. Animal Learning \& Behavior, 8, 231-236.

HollAND, P. C. (1985). The nature of conditioned inhibition in serial and simultaneous feature negative discriminations. In R. R. Miller \& N. E. Spear (Eds.), Information processing in animals: Conditioned inhibition (pp. 267- 297). Hillsdale, NJ: Erlbaum

HollaNd, P. C. (1989). Transfer of negative occasion setting and conditioned inhibition across conditioned stimuli and unconditioned stimuli. Journal of Experimental Psychology: Animal Behavior Processes, 15. 311-328.

Holland, P. C., GoRY, J. (1986). Extinction of inhibition after serial and simultaneous feature negative discrimination training. Quarterly Journal of Experimental Psychology, 38B, 245-265.

KoNORSk1, J. (1948). Conditioned reflexes and neuron organization. New York: Cambridge University Press.

KonORSKI, J. (1967). Insegrative activity of the brain. Chicago: University of Chicago Press.

LYSLE, D. T., Fowler, H. (1985). Inhibition as a "slave" process: Deactivation of conditioned inhibition through extinction of conditioned excitation. Journal of Experimental Psychology: Animal Behavior Processes, 11, 71-94.

Mazur, J. E., Wagner, A. R. (1982). An episodic model of associative learning. In $M$. Commons, R. Herrnstein, \& A. R. Wagner (Eds.), Quantitative analyses of behavior: Acquisition (Vol. 3, pp. 339). Cambridge, MA: Ballinger.

Pearce, J. M., Hall, G. (1980). A model for Pavlovian leaming: Variations in the effectiveness of conditioned but not of unconditioned stimuli. Psychological Review, 87, 532-552.

Pearce, J. M., Nicholas, D. J., Dickinson, A. (1982). Loss of associability by a conditioned inhibitor. Quarterly Joumal of Experimental Psychology, 33B, 149-162.

Rescort.A, R. A. (1979). Conditioned inhibition and extinction. In A. Dickinson \& R. A. Boakes (Eds.), Mechanisms of learning and moti- 
vation: A memorial volume for Jerzy Konorski (pp. 83-110). Hillsdale, NJ: Erlbaum.

Rescorla, R. A. (1982). Some consequences of associations between the excitor and the inhibitor in conditioned inhibition. Journal of $E x$ perimental Psychology: Animal Behavior Processes, 8, 288-298.

Rescorla, R. A. (1985). Conditioned inhibition and facilitation. In R. R. Miller \& N. E. Spear (Eds.), Information processing in animals: Conditioned inhibition (pp. 299-326). Hillsdale, NJ: Erlbaum.

ResCorlA, R. A. (1989). Simultaneous and sequential conditioned inhibition in autoshaping. Quarterly Journal of Experimental Psychology, 41B, 275-286.

RESCORLA, R. A. (1991). Separate reinforcement can enhance the effectiveness of modulators. Joumal of Experimental Psychology: Animal Behavior Processes, 17, 259-269.

Rescorla, R. A., \& WAgner, A. R. (1972). A theory of Pavlovian conditioning: Variations in the effectiveness of reinforcement and nonreinforcement. In A. H. Black \& W. F. Prokasy (Eds.), Classical conditioning II (pp. 64-99). New York: Appleton-Century-Crofts.

Robiss, S. J. (1990). Mechanisms underlying spontaneous recovery in autoshaping. Joumal of Experimental Psychology: Animal Behavior Processes, 16, 235-249.
WAGNER, A. R. (1978). Expectancies and the priming of STM. In S. H. Hulse, H. Fowler, \& W. K. Honig (Eds.), Cognitive aspects of animal behavior (pp. 177-209). Hillsdale, NJ: Erlbaum.

WAGNER, A. R. (1981). SOP: A model of automatic memory processing in animal behavior. In N. E. Spear \& R. R. Miller (Eds.), Information processing in animals: Memory mechanisms (pp. 5-47). Hillsdale, NJ: Erlbaum.

Wagner, A. R., Rescorla, R. A. (1972). Inhibition in Pavlovian conditioning: Application of a theory. In R. A. Boakes \& M. S. Halliday (Eds.), Inhibition and learning (301-336). London: Academic Press.

Witcher, E. S., A Ayres, J. J. B. (1984). A test of two methods for extinguishing Pavlovian conditioned inhibition. Animal Leaming \& Behavior, 12, 149-156.

Zimmer-Hart, C. L., Rescorla, R. A. (1974). Extinction of Pavlovian conditioned inhibition. Journal of Comparative \& Physiological Psychology, 86, 837-845.

(Manuscript received November 20, 1990; revision accepted for publication June 20, 1991.) 